\title{
Inertial effects in the anomalous dielectric relaxation of rotators in space
}

\author{
William T. Coffey \\ Department of Electronic and Electrical Engineering, Trinity College, Dublin 2, Ireland \\ Yuri P. Kalmykov \\ Centre d'Etudes Fondamentales, Université de Perpignan, 52 Avenue de Villeneuve, 66860 Perpignan Cedex, France \\ Sergey V. Titov \\ Institute of Radio Engineering and Electronics of the Russian Academy of Sciences, Vvedenskii Square 1, Fryazino, Moscow Region, \\ 141190, Russian Federation \\ (Received 4 December 2001; published 2 May 2002)
}

\begin{abstract}
The linear dielectric response of an assembly of noninteracting linear (needlelike) dipole molecules (each of which is free to rotate in space) is evaluated in the context of fractional dynamics. The infinite hierarchy of differential-recurrence relations for the relaxation functions appropriate to the dielectric response is derived by using the underlying inertial fractional Fokker-Planck (fractional Klein-Kramers) equation. On solving this hierarchy in terms of continued fractions (as in the normal rotational diffusion), the complex dynamic susceptibility is obtained and is calculated for typical values of the model parameters. It is shown that the model can reproduce nonexponential anomalous dielectric relaxation behavior at low frequencies $(\omega \tau \leqslant 1$, where $\tau$ is the Debye relaxation time) and the inclusion of inertial effects ensures that optical transparency is regained at very high frequencies (in the far infrared region) so that Gordon's sum rule for integral dipolar absorption is satisfied.
\end{abstract}

DOI: 10.1103/PhysRevE.65.051105

PACS number(s): 05.40.-a; 77.22.-d; 05.45.Df

\section{INTRODUCTION}

An extension of the Debye model [1] of dielectric relaxation of an assembly of noninteracting dipolar molecules to fractional dynamics for the purpose of providing a theoretical basis for the understanding of the phenomenon of the anomalous dielectric relaxation arising from diffusion processes in disordered fractal structures has recently been given by Coffey et al. [2]. The initial Debye treatment of the dielectric relaxation problem [1], although considering assemblies of both fixed axis and space rotators, does not include the inertial effects of the molecules and so is valid only in the low frequency or small inertial effects limit. The neglect of inertia in the dynamical processes then leads to the phenomenon of infinite dielectric absorption at high frequencies both in the fractional and in the conventional Brownian dynamics that arises from the normal diffusion in regular space. Both the Debye theory [1] and its subsequent extension to fractional dynamics are based on the Smoluchowski equation generalized to fractional dynamics $[2,3]$. Both are approximate equations for the evolution of the distribution function of the orientations of the dipoles in configuration space and exclude inertial effects. Thus, as in the original Einstein theory of Brownian motion, conclusions about the relaxation processes based on these equations will be invalid at short times, corresponding to high frequencies. In the frequency domain this will give rise to the absurd result of infinite integral absorption so that a return to optical transparency at very high frequencies is impossible. Hence, an accurate theory that ensures finite integral absorption and a return to optical transparency may only be constructed by using the Klein-Kramers equation or its fractional dynamics equivalent for the evolution of the distribution function in phase space. For rotation in space, the phase space comprises the angular momenta and Euler angles. Thus the present calculations also serve as a simple example of the solution of the fractional Klein-Kramers equation for a multiple degree of freedom system.

Mindful of the limitations imposed on the Debye theory and its fractional extension by using a diffusion equation of Smoluchowski type, Coffey et al. [4] have shown (by generalizing the approach given by Gross [5] and Sack [6,7], for normal rotational diffusion including inertial effects of fixed axis rotators) how inertial effects may be included in the fractional dynamics of an assembly of noninteracting fixed axis rotators. The starting point of the solution outlined in Ref. [4] is the generalization of the Klein-Kramers equation to fractional dynamics, originally proposed by Barkai and Silbey [9] for translational motion, as adapted to rotational motion about a fixed axis. The inclusion of inertial effects in the fractional dynamics just as in the conventional Brownian dynamics thus ensures the desired return to optical transparency at high frequencies. Moreover, Gordon's sum rule [8] for the integral dipolar absorption is satisfied. In addition, just as in normal diffusion [6], the complex susceptibility may be written in closed form in terms of Kummer's functions or as a continued fraction [4]. We have also demonstrated in Ref. [4] that this generalized model can reproduce nonexponential (Cole-Cole type) anomalous dielectric relaxation behavior, and how the unphysical high frequency behavior of the absorption coefficient due to the neglect of inertia may be removed in fractional relaxation as in inertia corrected Debye relaxation.

Although the fixed axis rotator model considered in Ref. 
[4] reproduces the principal features of dielectric relaxation of an ensemble of dipolar molecules and allows one considerable mathematical simplification of the problem [6,7], this model may be used only for the qualitative evaluation of dielectric spectra. The quantitative theory of dielectric relaxation requires an analysis of molecular reorientations in three dimensions [8]. In the present paper, we shall generalize the results of Ref. [4] and demonstrate how the analogous fractional Klein-Kramers equation (FKKE) pertaining to rotation in space may also be solved to yield the complex dielectric susceptibility in terms of continued fractions, thus extending the results of Sack [7] originally given for normal rotational diffusion in space including inertial effects to fractional dynamics. Our starting point will be the separation of variables method of solution of the FKKE coupled with a generalization of the integral theorem $[10,11]$ of Laplace transformation to fractional indices. We shall also confine ourselves to the simplest model of rotation in space, namely, an assembly of noninteracting needlelike dipolar molecules (treated originally by Sack [7] and later by McConnell [12], Coffey $[13,14]$, and Coffey et al. [15]). The results for the more complicated sphere model then follow, if desired, by analogy with the work of Sack [7] for normal diffusion. The aftereffect solution for the dynamic Kerr effect [16] may be treated in analogous fashion and is also presented.

\section{THE FRACTIONAL KLEIN-KRAMERS EQUATION FOR THE NEEDLE MODEL}

Let us consider the rotational motion of a thin rod, or rotator, representing the polar molecule, which is subjected to an external electric field $\mathbf{E}[7,12]$. We assume that the field $\mathbf{E}$ is parallel to the $Z$ axis of the laboratory coordinate system $O X Y Z$. In the molecular coordinate system oxyz rigidly connected to the rotator, the components of the angular velocity $\boldsymbol{\omega}$ of the rotator and of the torques $\mathbf{K}$ produced by the field $\mathbf{E}$ are [12]

$$
\begin{gathered}
\boldsymbol{\omega}=\left(\omega_{x}, \omega_{y}, \omega_{z}\right)=(\dot{\vartheta}, \dot{\varphi} \sin \vartheta, \dot{\varphi} \cos \vartheta), \\
\mathbf{K}=(-\mu E \sin \vartheta, 0,0),
\end{gathered}
$$

where $\vartheta(t)$ and $\varphi(t)$ are the polar and azimuthal angles, and $\boldsymbol{\mu}$ is the dipole moment of the rotator. Here, the internal field effects are ignored, which means that the effects of longrange torques due to the interaction between the average moments and the Maxwell fields are not taken into account. Such effects may be discounted for dilute systems in first approximation. Thus, the results obtained here are relevant to situations where dipole-dipole interactions have been eliminated by extrapolation of data to infinite dilution.

In order to describe the fractional Brownian rotational motion, we use the FKKE for the evolution of the probability density function $W$ in configuration-angular-velocity space for linear molecules in the same form as in Ref. [4] for fixed axis rotators (this form of the FKKE was suggested by Barkai and Silbey [9] for one-dimensional translational Brownian motion). For rotators in space, the FKKE becomes

$$
\begin{gathered}
\frac{\partial W}{\partial t}+\omega_{x} \frac{\partial W}{\partial \theta}+\omega_{y} \cot \vartheta\left(\omega_{y} \frac{\partial W}{\partial \omega_{x}}-\omega_{x} \frac{\partial W}{\partial \omega_{y}}\right) \\
-\frac{\mu E}{I} \sin \vartheta \frac{\partial W}{\partial \omega_{x}}={ }_{0} D_{t}^{1-\alpha} L_{\mathrm{FP}} W,
\end{gathered}
$$

where

$$
\begin{aligned}
{ }_{0} D_{t}^{1-\alpha} L_{\mathrm{FP}} W= & { }_{0} D_{t}^{1-\alpha} \Theta \beta\left[\frac{\partial}{\partial \omega_{x}}\left(\omega_{x} W+\frac{k_{B} T}{I} \frac{\partial W}{\partial \omega_{x}}\right)\right. \\
& \left.+\frac{\partial}{\partial \omega_{y}}\left(2 \omega_{y} W+\frac{k_{B} T}{I \cos ^{2} \vartheta} \frac{\partial W}{\partial \omega_{y}}\right)\right]
\end{aligned}
$$

is the fractional Fokker-Planck operator, $\beta=\zeta / I, \zeta$ is the viscous damping coefficient of a dipole, $k_{B} T$ is the thermal energy, $I$ is the moment of inertia of the rotator about the axis of rotation, $\Theta=\tau^{1-\alpha}, \tau$ is the intertrapping time scale which we identify with the Debye relaxation time $\zeta /\left(2 k_{B} T\right)$ for linear molecules, and $\alpha$ is the exponent characterizing the anomalous diffusion process. The operator ${ }_{0} D_{t}^{1-\alpha}$ $\equiv(\partial / \partial t)_{0} D_{t}^{-\alpha}$ in Eq. (4) is defined in terms of the convolution (the Riemann-Liouville fractional integral definition) [3]

$$
{ }_{0} D_{t}^{-\alpha} W(, t)=\frac{1}{\Gamma(\alpha)} \int_{0}^{t} \frac{W\left(, t^{\prime}\right) d t^{\prime}}{\left(t-t^{\prime}\right)^{1-\alpha}}
$$

so that the fractional derivative is a type of memory function $[3,8]$ or stosszahlansatz for the Boltzmann equation $[17,18]$ underlying the FKKE. For $\alpha=1$, the fractional FokkerPlanck operator of Eq. (4) reduces to that corresponding to normal inertia corrected rotational diffusion considered by Sack [7]. Just as for $\alpha=1$, Eq. (3) is independent of the azimuthal angle $\varphi$ and the $z$ component of the angular velocity $\omega_{z}$ so that for the problem in question one may ignore the dependence of $W$ on $\varphi$ and $\omega_{z}$.

\section{CONTINUED FRACTION SOLUTION FOR DIELECTRIC RELAXATION}

Let us suppose that the uniform field $\mathbf{E}$, having been applied to the assembly of dipoles at a time $t=-\infty$ so that equilibrium conditions prevail by the time $t=0$, is switched off at $t=0$. In addition it is supposed that the field is weak (i.e., $\mu E \ll k_{B} T$, which is the linear response condition). We seek a solution of the FKKE, Eq. (3), for the case $E=0$ at $t>0$ by using the method of separation of variables in the form of the series

$$
\begin{aligned}
W\left(\vartheta, \omega_{x}, \omega_{y}, t\right)= & \eta^{2} e^{-\eta^{2}\left(\omega_{x}^{2}+\omega_{y}^{2}\right)} \sum_{l=0}^{\infty} \sum_{m=0}^{l} \sum_{n=0}^{\infty} a_{n}^{l, m} c_{n}^{l, m}(t) \\
& \times s_{n}^{m}\left(\omega_{x}, \omega_{y}\right) P_{l}^{m}(\cos \vartheta)
\end{aligned}
$$

where

$$
c_{n}^{l, m}(t)=\left\langle s_{n}^{m}\left(\omega_{x}, \omega_{y}\right) P_{l}^{m}(\cos \vartheta)\right\rangle,
$$


$P_{l}^{m}(z)$ are the associated Legendre functions of order $l(m$ $\leqslant l)[19], \eta=\sqrt{I /\left(2 k_{B} T\right)}$, and the angular brackets \langle\rangle denote ensemble averages over the distribution function $W$, viz.,

$$
\begin{aligned}
\langle A\rangle(t)= & \int_{-\infty}^{\infty} \int_{-\infty}^{\infty} \int_{0}^{2 \pi} A\left(\vartheta, \omega_{x}, \omega_{y}\right) W\left(\vartheta, \omega_{x}, \omega_{y}, t\right) \\
& \times \sin \vartheta d \vartheta d \omega_{x} d \omega_{y} .
\end{aligned}
$$

The orthogonal functions $s_{n}^{m}\left(\omega_{x}, \omega_{y}\right)$ are given in terms of finite series of products of Hermite polynomials $H_{n}$ [19] in the components of the angular velocity as

$$
\begin{aligned}
s_{n}^{2 m+M}\left(\omega_{x}, \omega_{y}\right)= & \sum_{q=0}^{n} \frac{r_{2 m+M}(n, q)}{q !(n-q) !} H_{2 n-2 q+M}\left(\eta \omega_{x}\right) \\
& \times H_{2 q}\left(\eta \omega_{y}\right) \quad(M=0,1),
\end{aligned}
$$

where the coefficients $r_{2 m+M}(n, q)$ can be determined from the following recurrence relations:

$$
\begin{aligned}
r_{2 m}(n, q)= & \left(n-q+\frac{1}{2}\right)\left(1-\frac{2 q+1}{2 m-1}\right) r_{2 m-1}(n, q) \\
& +(n-q) \frac{2 q+1}{2 m-1} r_{2 m-1}(n, q+1), \\
r_{2 m+1}(n, q)= & \left(1+\frac{q}{m}\right) r_{2 m}(n, q)-\frac{q}{m} r_{2 m}(n, q-1)
\end{aligned}
$$

with $r_{0}(n, q)=r_{1}(n, q)=1$ [for example, $r_{2}=n-2 q, r_{3}=n$ $-4 q, r_{4}=n(n-1)-8 q(n-q)$, etc.]. The normalizing constants $a_{n}^{l, m}$ are obtained by means of the orthogonality properties of the $P_{l}^{m}(\cos \vartheta)$ and $H_{n}(z)$, viz. [19],

$$
\begin{gathered}
\int_{0}^{\pi} P_{l}^{m}(\cos \vartheta) P_{l^{\prime}}^{m^{\prime}}(\cos \vartheta) \sin \vartheta d \vartheta \\
=\frac{2(l+m) !}{(2 l+1)(l-m) !} \delta_{l, l^{\prime}} \delta_{m, m^{\prime}}, \\
\int_{-\infty}^{\infty} H_{n}(x) H_{n^{\prime}}(x) e^{-x^{2}} d x=\sqrt{\pi} 2^{n} n ! \delta_{n, n^{\prime}}
\end{gathered}
$$

so that

$$
\left(a_{n}^{l, m}\right)^{-1}=\frac{(l+m) ! b_{n}^{m}}{\pi(2 l+1)(l-m) ! 2^{4 n-1}},
$$

where $b_{n}^{0}=1, b_{n}^{1}=1 /[2(n+1)], b_{n}^{2}=1 / n^{2}, \ldots$. Our separation of the variables procedure based on Eq. (6) differs from that introduced by Sack [7] and allows us to solve the FKKE in a simpler manner.

Substituting Eq. (6) into the FKKE Eq. (3), taking the inner product, and utilizing the orthogonal properties and known recurrence relations [19] for the associated Legendre functions $P_{l}^{m}(\cos \vartheta)$, Eq. (10), and the Hermite polynomials
$H_{n}(z)$, Eq. (11), then yields an infinite hierarchy of differential recurrence relations for the $c_{n}^{l, m}(t)$ governing the orientational relaxation of the system, viz.,

$$
\eta \frac{d}{d t} c_{n}^{l, 0}(t)=-2 n \eta_{0} D_{t}^{1-\alpha} \Theta \beta c_{n}^{l, 0}(t)+\frac{1}{2} c_{n}^{l, 1}(t)+2 c_{n-1}^{l, 1}(t),
$$

$$
\begin{aligned}
\eta \frac{d}{d t} c_{n}^{l, 1}(t)= & -(2 n+1) \eta_{0} D_{t}^{1-\alpha} \Theta \beta c_{n}^{l, 1}(t)+c_{n}^{l, 2}(t) \\
& +\frac{1}{4} c_{n+1}^{l, 2}(t)-l(l+1)(n+1) \\
& \times\left[c_{n}^{l, 0}(t)+\frac{1}{4} c_{n+1}^{l, 0}(t)\right] \\
\eta \frac{d}{d t} c_{n}^{l, 2}(t)= & -2 n \eta_{0} D_{t}^{1-\alpha} \Theta \beta c_{n}^{l, 2}(t)-\frac{(l+2)(l-1) n}{4} c_{n}^{l, 1}(t) \\
& -(l+2)(l-1)(n+1) c_{n-1}^{l, 1}(t)+c_{n-1}^{l, 3}(t) \\
& +\frac{1}{4} c_{n}^{l, 3}(t),
\end{aligned}
$$

and so on.

In dielectric relaxation $l=1$ so that by taking the Laplace transform of Eqs. (12)-(14) over the time variables and noting the generalized integral theorem for Laplace transforms $[3,10,11]$, namely,

$$
L\left\{{ }_{0} D_{t}^{1-\alpha} f(t)\right\}=\left\{\begin{array}{l}
s^{1-\alpha} \widetilde{f}(s)-\left.{ }_{0} D_{t}^{-\alpha} f(t)\right|_{t=0} \quad(0<\alpha<1) \\
s^{1-\alpha} \widetilde{f}(s) \quad(1 \leqslant \alpha<2),
\end{array}\right.
$$

where $\widetilde{f}(s)=L\{f(t)\}=\int_{0}^{\infty} e^{-s t} f(t) d t$, we then have a system of algebraic recurrence relations for the Laplace transform of $c_{n}^{1, m}(t)(m=0,1)$ governing the dielectric response, namely,

$$
\begin{gathered}
{[\eta s+2 n x] \widetilde{c}_{n}^{1,0}(s)-2 \widetilde{c}_{n-1}^{1,1}(s)-\widetilde{c}_{n}^{1,1}(s) / 2=\delta_{n, 0} \eta c_{0}^{1,0}(0),} \\
{[\eta s+(2 n+1) x] \widetilde{c}_{n}^{1,1}(s)+2(n+1)\left[\widetilde{c}_{n}^{1,0}(s)+\widetilde{c}_{n+1}^{1,0}(s) / 4\right]=0 .}
\end{gathered}
$$

Here $x=\gamma^{\prime}(\tau s)^{1-\alpha} / 2, \gamma^{\prime}=\zeta \sqrt{2 / I k_{B} T}$ is the inertial effects parameter (large $\gamma^{\prime}$ corresponds to small inertial effects and vice versa), and we have taken into account that all the $c_{n}^{1,0}(0)$ vanish with the exception of $n=0$, viz., $c_{0}^{1,0}(0)$ $=\xi / 3$, where $\xi=\mu E /\left(k_{B} T\right)$. The last equality follows from the linearized initial (at $t=0$ ) distribution function, which has the Maxwell-Boltzmann form

$$
\begin{aligned}
W\left(\vartheta, \omega_{x}, \omega_{y}, 0\right)= & \frac{1}{2 \pi} \eta^{2} e^{-\eta^{2}\left(\omega_{x}^{2}+\omega_{x}^{2}\right)} \\
& \times\left(1+\xi \cos \vartheta+\frac{\xi^{2}}{4 \pi^{2}} \cos ^{2} \vartheta\right)+o\left(\xi^{2}\right)
\end{aligned}
$$


The solution of Eqs. (16) and (17) can obtained as a scalar continued fraction as follows. First of all, Eqs. (16) and (17) can be rearranged to yield

$$
\left(\eta s-q_{n}\right) \widetilde{c}_{n}^{1,0}(s)-q_{n}^{+} \widetilde{c}_{n+1}^{1,0}(s)-q_{n}^{-} \widetilde{c}_{n-1}^{1,0}(s)=\delta_{n, 0} \eta c_{0}^{1,0}(0),
$$

where

$$
q_{n}=-\frac{n}{(2 n-1) x+\eta s}-2 n x-\frac{(n+1)}{(2 n+1) x+\eta s},
$$

$$
q_{n}^{+}=-\frac{(n+1) / 4}{(2 n+1) x+\eta s},
$$

$$
q_{n}^{-}=-\frac{4 n}{(2 n-1) x+\eta s} .
$$

Equation (19) can now be solved using the continued fraction

$$
\tilde{c}_{0}^{1,0}(s)=\frac{\eta c_{0}^{1,0}(0)}{\eta s-q_{0}-\frac{q_{0}^{+} q_{1}^{-}}{\eta s-q_{1}-\frac{q_{1}^{+} q_{2}^{-}}{\eta s-q_{2}-\cdots}}}
$$

Equation (20) can be further rearranged to yield

$$
\frac{\widetilde{c}_{n}^{1,0}(s)}{c_{0}^{1,0}(0)}=\frac{\eta}{\eta s+\frac{1}{x+\eta s+\frac{1}{2 x+\eta s+\frac{2}{3 x+\eta s+\frac{3 x+\eta s+\frac{3}{5 x+\eta s+\cdots}}{4 x+\cdots}}}}}
$$

An alternative approach to the evaluation of $\widetilde{c_{0}^{1,0}}(s)$ in terms of matrix continued fractions [14] is presented in Appendix A.

Having determined $\widetilde{c}_{0}^{1,0}(s)=\overline{\left\langle P_{1}(\cos \vartheta\right.}(s)$, one can calculate the normalized complex susceptibility $\hat{\chi}(\omega)$ $=\chi(\omega) / \chi^{\prime}(0)\left[\chi^{\prime}(0)=\mu^{2} N /\left(3 k_{B} T\right)\right.$ is the static susceptibility and $N$ is the number of dipoles per unit volume], which is given by linear response theory as [20]

$$
\hat{\chi}(\omega)=\hat{\chi}^{\prime}(\omega)-i \hat{\chi}^{\prime \prime}(\omega)=1-i \omega \frac{\widetilde{c}_{0}^{1,0}(i \omega)}{c_{0}^{1,0}(0)} .
$$

Here, the quantity $\widetilde{c}_{0}^{1,0}(i \omega) / c_{0}^{1,0}(0)$ coincides with the onesided Fourier transform of the normalized dipole autocorrelation function $C_{1}(t)=\langle\cos \vartheta(0) \cos \vartheta(t)\rangle_{0} /\left\langle\cos ^{2} \vartheta(0)\right\rangle_{0}$, namely,

$$
\frac{\widetilde{c}_{0}^{1,0}(i \omega)}{c_{0}^{1,0}(0)}=\int_{0}^{\infty} C_{1}(t) e^{-i \omega t} d t
$$

The zero on the angular brackets denotes that the ensemble average is taken in the absence of the field.
The approach developed may also be extended to treat all the other averages $\left\langle P_{n}(\cos \vartheta)\right\rangle(t)$ characterizing orientational relaxation in fluids [8]. In particular, the evaluation of the average of the second order Legendre polynomial $\left\langle P_{n}(\cos \vartheta)\right\rangle(t)$ (this quantity describes the dynamic Kerreffect [16]) is given in Appendix B.

\section{RESULTS AND DISCUSSION}

The infinite continued fraction Eq. (21) is very convenient for the purpose of calculations so that the complex dielectric susceptibility Eq. (22) can be readily evaluated for all values of the model parameters $\eta, \gamma^{\prime}$, and $\alpha$. For $\alpha=1$, the anomalous rotational diffusion solution Eq. (21) coincides with that of Sack [7] for normal rotational diffusion [in order to obtain a complete agreement, one has to introduce Sack's inertial parameter $\gamma=2 / \gamma^{\prime 2}$ and to carry out elementary mathematical transformations in Eq. (21)]. Moreover, in a few particular cases, Eqs. (21) and (22) can be considerably simplified. In the free rotation limit $(\zeta=0)$, which corresponds to the continued fraction Eq. (21) evaluated at $x=0$, that fraction can be expressed (just as for normal rotational diffusion [7]) in terms of the exponential integral function $E_{1}(z)$ [19] so that the normalized complex susceptibility is

$$
\hat{\chi}(\omega)=1+\eta^{2} \omega^{2} e^{-\eta^{2} \omega^{2}} E_{1}\left(-\eta^{2} \omega^{2}\right) .
$$




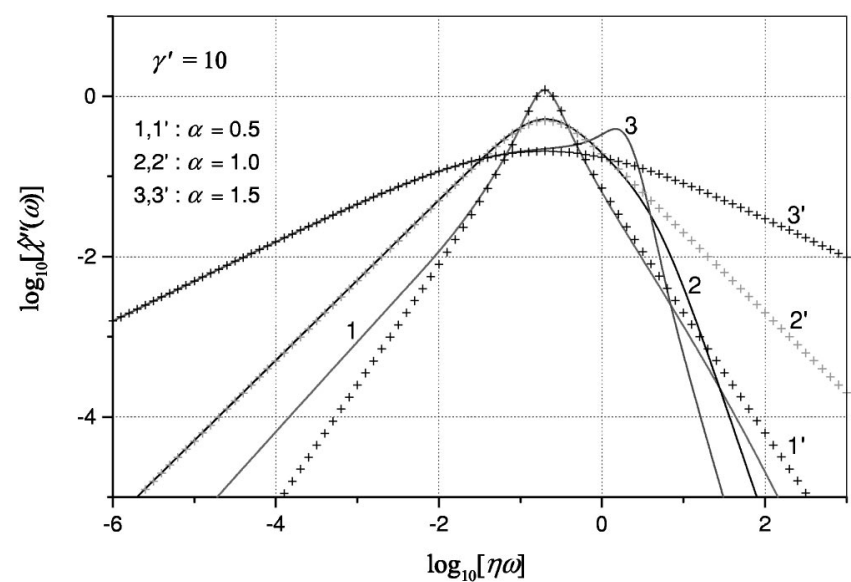

FIG. 1. Dielectric loss spectra $\hat{\chi}^{\prime \prime}(\omega)$ for $\gamma^{\prime}=10$ and various values of $\alpha . \alpha=0.5$ (curves 1 and $1^{\prime}$ ), 1.0 (curves 2 and $2^{\prime}$ ), and 1.5 (curves 3 and $3^{\prime}$ ). Solid lines (1, 2, and 3), Eqs. (21) and (22); crosses $\left(1^{\prime}, 2^{\prime}\right.$, and $\left.3^{\prime}\right)$, Eq. (26).

Furthermore, just as in the one degree of freedom fixed axis rotation model [4], in the high damping limit $\left(\gamma^{\prime} \gg 1\right)$, Eq. (22) can be simplified yielding the generalization to fractional dynamics of the Rocard [16] equation, namely,

$$
\hat{\chi}(\omega)=\frac{1}{1+(i \omega \tau)^{\sigma}-(\omega \eta)^{2}},
$$

where $\sigma=2-\alpha$. On neglecting inertial effects $(\eta \rightarrow 0)$, Eq. (25) becomes

$$
\hat{\chi}(\omega)=\frac{1}{1+(i \omega \tau)^{\sigma}},
$$

i.e., the result previously proposed from empirical considerations [21,22]. For $\sigma=1$, Eq. (26) reduces to the Debye equation [1].

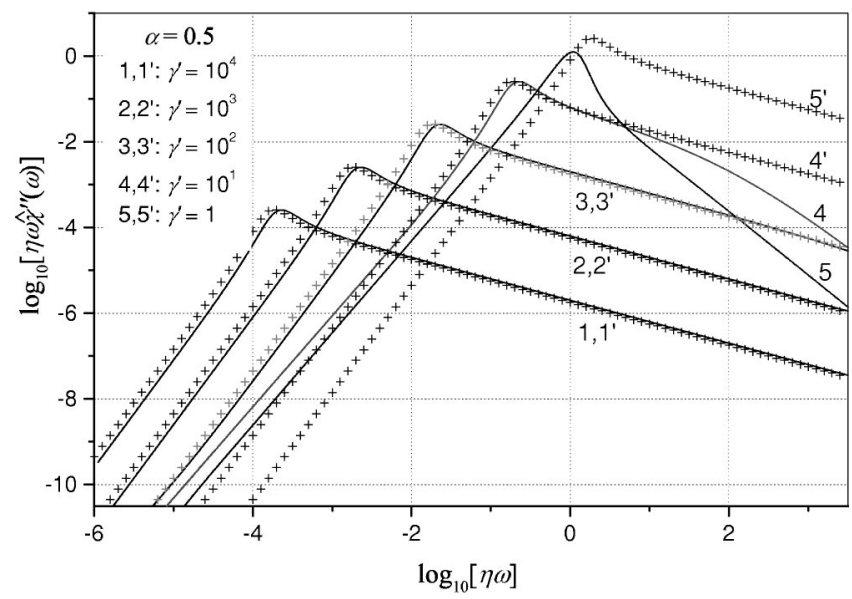

FIG. 2. Dielectric absorption spectra $\omega \hat{\chi}^{\prime \prime}(\omega)$ for $\alpha=0.5$ (enhanced diffusion) and various values of $\gamma^{\prime}: \gamma^{\prime}=10^{4}$ (curves 1 and $1^{\prime}$ ), $10^{3}$ (curves 2 and $2^{\prime}$ ), $10^{2}$ (curves 3 and $3^{\prime}$ ), 10 (curves 4 and $4^{\prime}$ ), and 1 (curves 5 and $5^{\prime}$ ). Solid lines (1, 2, 3, 4, and 5), Eqs. (21) and (22); crosses $\left(1^{\prime}, 2^{\prime}, 3^{\prime}, 4^{\prime}\right.$, and $\left.5^{\prime}\right)$, Eq. (26).

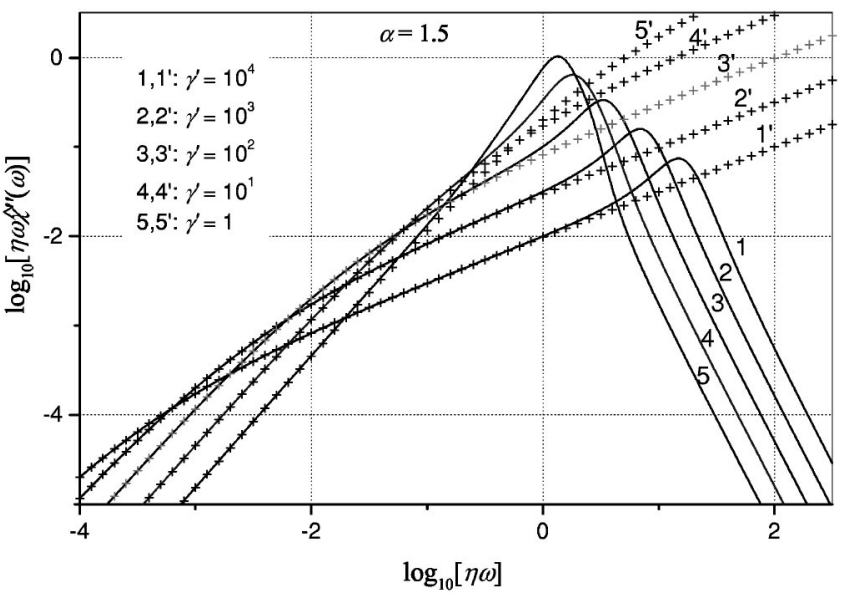

FIG. 3. The same as in Fig. 2 for $\alpha=1.5$ (subdiffusion).

Dielectric loss $\hat{\chi}^{\prime \prime}(\omega)$ and absorption $\omega \hat{\chi}^{\prime \prime}(\omega)$ spectra for various values of $\alpha$ and $\gamma^{\prime}$ are shown in Figs. 1-3. The Cole-Cole plot $\left[\hat{\chi}^{\prime \prime}(\omega)\right.$ vs $\left.\hat{\chi}^{\prime}(\omega)\right]$ is presented in Fig. 4. It is apparent that the half-width and the shape of the dielectric spectra strongly depend on both $\alpha$ (which in the present context pertains to anomalous diffusion in velocity space) and $\gamma^{\prime}$ (which characterizes the effects of molecular inertia). In the high damping limit $\left(\gamma^{\prime} \gg 1\right)$ and for $\alpha>1$ corresponding to $\sigma<1$ (subdiffusion in configuration space), the low frequency part of $\hat{\chi}^{\prime \prime}(\omega)$ may be approximated by the modified Debye equation (26). On the other hand, the high frequency behavior of $\hat{\chi}^{\prime \prime}(\omega)$ is entirely determined by the inertia of the system. For a given value of $\gamma^{\prime}$, the inertial effects become more pronounced when $\alpha \rightarrow 2$ (see Fig. 4). Just as in Brownian dynamics, it is apparent that inertial effects produce a much more rapid falloff of $\hat{\chi}^{\prime \prime}(\omega)$ at high frequencies. One can show that the fractional model under consideration satisfies the Gordon sum rule for the dipole integral absorption of rotators in space [23], viz.,

$$
\int_{0}^{\infty} \omega \chi^{\prime \prime}(\omega) d \omega=\frac{\pi N \mu^{2}}{3 I} .
$$

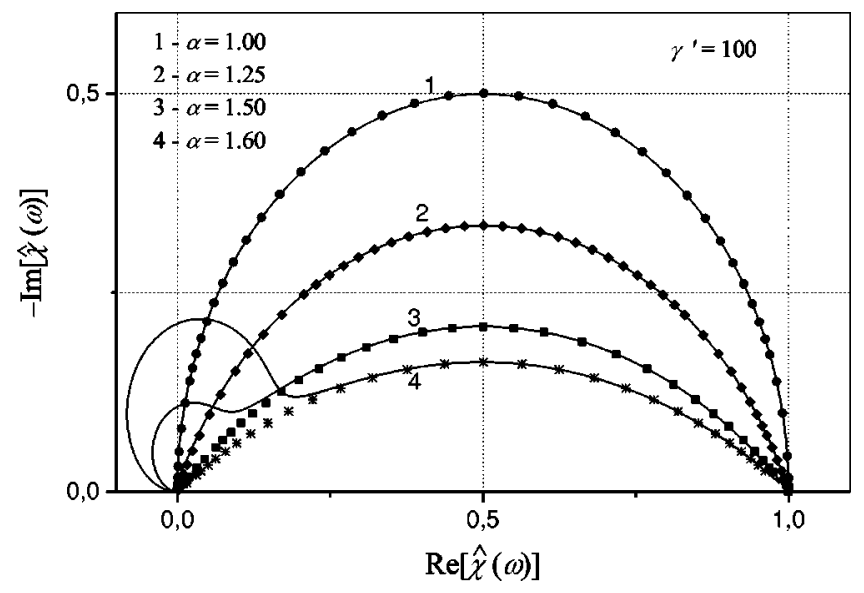

FIG. 4. The Cole-Cole plot for $\gamma^{\prime}=100$ and various $\alpha$ (subdiffusion): $\alpha=1$ (curve 1), 1.25 (curve 2), 1.5 (curve 3), and 1.6 (curve 4). Solid lines (1, 2, 3, and 4), Eqs. (21) and (22); symbols, Eq. (26). 
TABLE I. Comparison of the results for fixed axis rotators and rotators in space.

\begin{tabular}{|c|c|c|}
\hline & Fixed axis rotators [4] & Rotators in space \\
\hline $\begin{array}{l}\text { Characteristic } \\
\text { relaxation time }\end{array}$ & $\tau=\zeta /\left(k_{B} T\right)=\eta \gamma^{\prime}$ & $\tau=\zeta /\left(2 k_{B} T\right)=\eta \gamma^{\prime} / 2$ \\
\hline $\begin{array}{c}\text { Static } \\
\text { susceptibility }\end{array}$ & $\chi^{\prime}(0)=\mu^{2} N /\left(2 k_{B} T\right)$ & $\chi^{\prime}(0)=\mu^{2} N /\left(3 k_{B} T\right)$ \\
\hline Generalized & 1 & 1 \\
\hline Rocard equation & $\hat{\chi}(\omega)=\overline{1+(i \omega \tau)^{\sigma}-2(\omega \eta)^{2}}$ & $\hat{\chi}(\omega)=\overline{1+(i \omega \tau)^{\sigma}-(\omega \eta)^{2}}$ \\
\hline Gordon's sum rule & $\int_{0}^{\infty} \omega \chi^{\prime}(\omega) d \omega=\frac{\pi N \mu^{2}}{4 I}$ & $\int_{0}^{\infty} \omega \chi^{\prime \prime}(\omega) d \omega=\frac{\pi N \mu^{2}}{3 I}$ \\
\hline $\begin{array}{c}\text { Dielectric loss at } \zeta=0 \\
(\text { free rotation limit })\end{array}$ & $\hat{\chi}_{\mathrm{FR}}^{\prime \prime}(\omega)=\sqrt{\pi} \eta \omega e^{-\eta^{2} \omega^{2}}$ & $\hat{\chi}_{\mathrm{FR}}^{\prime \prime}(\omega)=\pi \eta^{2} \omega^{2} e^{-\eta^{2} \omega^{2}}$ \\
\hline
\end{tabular}

It is significant that the right hand side of Eq. (27) is determined by molecular parameters only and is independent of the temperature and the model parameters $\alpha$ and $\zeta$. In contrast, the fractional Debye model does not predict the correct value of the integral absorption: e.g., for $\alpha>1$, it predicts infinite integral absorption (see Fig. 3).

The behavior of the dielectric spectra for the two rotational degree of freedom (needle) model is similar but not identical to that for fixed axis rotators (one rotational degree of freedom model) [4]. Here, the one and two rotational degree of freedom models (fractional or normal) predict dielectric parameters that may considerably differ from each other. The differences in the results predicted by these two models are summarized in Table I. Here, one can readily see that the model of rotational Brownian motion of a fixed axis rotator treated in Ref. [4] only qualitatively reproduces the principal features (return to optical transparency, etc.) of dielectric relaxation of dipolar molecules in space, for example, the dielectric relaxation time obtained in the context of these models differs by a factor of 2 .

The result we have obtained for the complex susceptibility is of particular interest in the theory of dielectrics as it demonstrates how the unphysical high frequency divergence of the absorption coefficient in the far infrared region due to the neglect of inertia may be removed in fractional relaxation just as in inertia corrected Debye relaxation (see Figs. 1-3). We remark that the advantage of using the generalized integral theorem of Laplace transformation combined with continued fraction methods is that solutions for the complex susceptibility, etc., may be easily obtained, to any desired degree of accuracy, by elementary algebra manipulation without using special functions. Moreover, the generalized integral theorem indicates how existing normal diffusion solutions may easily be extended to include anomalous diffusion, which is again of particular interest in simplifying the problem of solution of the FKKE for multiple degree of freedom systems. We further remark that the continued fraction solutions that we have given, with a few elementary modifications, also yield the Laplace transform of the characteristic function of the configuration space distribution function including inertial effects. Thus all desired statistical averages such as the mean square angular displacement, etc., may be simply calculated by differentiation.
To conclude, we have demonstrated how conventional Brownian motion solutions for dielectric relaxation [7] may be generalized to fractional dynamics simply by using the generalized integral theorem of Laplace transformation [Eq. (15)] combined with the existing continued fraction solution, so providing one with a rigorous method of treating the rotational diffusion in disordered fractal systems. These systems generally have a waiting time probability density function, which governs the random time intervals between microscopic reorientations. Moreover, unlike the classical theory of Brownian motion, which has a characteristic microscopic time scale, namely, the mean duration of an elementary step in the underlying random walk (here microscopic reorientations), the characteristic waiting time is divergent as the system may maintain itself in a given orientation for an arbitrarily long time period. In other words, one is dealing with a fractal time random walk [24]. A general characteristic of the systems we have treated is that they are nonlocal in time and exhibit memory effects which give rise to anomalous rotational diffusion. For a further discussion couched in terms of the Langevin equation we refer the readers to a recent paper of Lutz [25], where anomalous relaxation of a free particle moving along the $x$-axis is discussed. This problem is the fractional dynamics equivalent of the Uhlenbeck-Ornstein process [26]. The present calculation also constitutes a good example of the solution of the FKKE for a multipledegree of freedom system and is to our knowledge the first example of such a solution. The methods we have outlined are also of importance when extended to other relaxation models such as the itinerant oscillator [27], which attempt to incorporate both resonance and relaxation behavior in a single model for the purpose of simultaneously explaining the Debye (low frequency) and far infrared absorption spectra of complex dipole systems.

\section{ACKNOWLEDGMENTS}

The support of this work by the Enterprise Ireland Research Collaboration Fund, USAF, EOARD (Contract No. F61773-01-WE407), the CNRS Enterprise Ireland-France award scheme, and the Russian Foundation for Basic Research (Project No. 01-02-16050) is gratefully acknowledged. Y.P.K. and S.V.T. thank the Royal Irish Academy for financial 
support. W.T.C. acknowledges support from the Berkeley Foundation TCD.

\section{APPENDIX A: SOLUTION OF EQS. (16) AND (17) IN TERMS OF MATRIX CONTINUED FRACTIONS}

Equations (16) and (17) can also be solved in terms of matrix continued fractions [5,14]. This is accomplished as follows. Let us introduce the column vectors

$$
\widetilde{\mathbf{C}}_{n}(s)=\left(\begin{array}{l}
\widetilde{c}_{n-1}^{1,0}(s) \\
\widetilde{c}_{n-1}^{1,1}(s)
\end{array}\right) \quad(n \geqslant 1) .
$$

Then the scalar recurrence relations (16) and (17) may be recast in the form of the matrix recurrence relations

$$
\begin{aligned}
& \left(\eta s-\mathbf{Q}_{n}\right) \widetilde{\mathbf{C}}_{n}(s)-\mathbf{Q}_{n}^{+} \widetilde{\mathbf{C}}_{n+1}(s)-\mathbf{Q}_{n}^{-} \widetilde{\mathbf{C}}_{n-1}(s) \\
& =\delta_{1, n} \eta \mathbf{C}_{1}(0) \quad(n \geqslant 1),
\end{aligned}
$$

where

$$
\begin{aligned}
& \mathbf{Q}_{n}^{-}=\left(\begin{array}{ll}
0 & 2 \\
0 & 0
\end{array}\right), \quad \mathbf{Q}_{n}=\left(\begin{array}{cc}
-2(n-1) x & 1 / 2 \\
-2 n & -(2 n-1) x
\end{array}\right), \\
& \mathbf{Q}_{n}^{+}=\left(\begin{array}{cc}
0 & 0 \\
-n / 2 & 0
\end{array}\right),
\end{aligned}
$$

and

$$
\mathbf{C}_{1}(0)=\left(\begin{array}{c}
c_{0}^{1,0}(0) \\
0
\end{array}\right)
$$

Equation (A2) has the solution

$$
\widetilde{\mathbf{C}}_{1}(s)=\eta \frac{\mathbf{I}}{\eta s \mathbf{I}-\mathbf{Q}_{1}-\mathbf{Q}_{1}^{+} \frac{\mathbf{I}}{\eta s \mathbf{I}-\mathbf{Q}_{2}-\mathbf{Q}_{2}^{+} \frac{\mathbf{I}}{\eta s \mathbf{I}-\mathbf{Q}_{3} \ldots} \mathbf{Q}_{3}^{-}} \mathbf{Q}_{2}^{-}} \mathbf{C}_{1}(0)
$$

where the fraction lines denote the matrix inversion. The calculation shows that the matrix continued fraction solution rendered by Eqs. (A3) and the ordinary continued fraction solutions Eq. (21) coincide.

\section{APPENDIX B: THE DYNAMIC KERR-EFFECT RESPONSE}

The physical quantity of interest from an experimental point of view and which is appropriate to Kerr effect relaxation is the electric birefringence function $K(t)$ defined by [16]

$$
K(t)=B_{2} \frac{2 \pi N_{0}}{\bar{n}}\left(\alpha_{\|}^{0}-\alpha_{\perp}^{0}\right)\left\langle P_{2}(\cos \vartheta)\right\rangle(t)
$$

where $N_{0}$ denotes the number of molecules per unit volume, $\alpha_{\|}^{0}$ and $\alpha_{\perp}^{0}$ are the components of the optical polarizability due to the electric field (optical frequency) of the light beam passing through the liquid medium, and $\bar{n}$ is the mean refractive index. The coefficient $B_{2}$ depends on the particle depolarization factors and the dielectric susceptibility of the medium.

In the transient (step-off) Kerr effect response, it is also possible to obtain from Eqs. (12)-(14) for $l=2$ the system of recurrence equations for the Laplace transforms of the corresponding relaxation functions $c_{n}^{2, m}(t)(m=0,1,2)$ pertaining to that response, viz.,

$$
[\eta s+2 n x] \widetilde{c}_{n}^{2,0}(s)-\frac{1}{2} \widetilde{c}_{n}^{2,1}(s)-2 \widetilde{c}_{n-1}^{2,1}(s)=\delta_{n, 0} \eta c_{0}^{2,0}(0),
$$

$$
\begin{aligned}
& {[s \eta+(2 n+1) x] \widetilde{c}_{n}^{2,1}(s)+\frac{3(n+1)}{2} \widetilde{c}_{n+1}^{2,0}(s)} \\
& \quad+6(n+1) \widetilde{c}_{n}^{2,0}(s)-\widetilde{c}_{n}^{2,2}(s)-\frac{1}{4} \widetilde{c}_{n+1}^{2,2}(s)=0,
\end{aligned}
$$

$$
[s \eta+2 n x] \widetilde{c}_{n}^{2,2}(s)+n \widetilde{c}_{n}^{2,1}(s)+4(n+1) \widetilde{c}_{n-1}^{2,1}(s)=0 .
$$

Here, we have taken into account that all the $c_{n}^{2, m}(0)$ vanish with the exception of $n=0$ and $m=0$, viz., $c_{0}^{2,0}(0)=\xi^{2} / 15$. This follows from the initial Maxwell-Boltzmann distribution Eq. (18).

Just as in the dielectric response, Eqs. (B1)-(B3) can be rearranged as a three-term recurrence equation for $\widetilde{c}_{n}^{2,1}(s)$ so that the exact solution for $\widetilde{c}_{0}^{2,1}(s)$ in terms of an infinite continued fraction combined with the relation

$$
s \widetilde{c}_{0}^{2,0}(s)=c_{0}^{2,0}(0)+\frac{1}{2 \eta} \widetilde{c}_{0}^{2,1}(s)
$$

is [that is, Eq. (B1) at $n=0$ ], 


$$
\frac{\widetilde{c}_{0}^{2.0}(s)}{c_{0}^{2,0}(0)}=\frac{\eta}{\eta s+\frac{3}{\eta s+x+\frac{5}{\eta s+2 x}-\frac{b_{0}}{\eta s+a_{1}-\frac{b_{1}}{\eta s+a_{2}-\frac{b_{2}}{\eta s+a_{3}-\cdots}}}}}
$$

where

$$
a_{n}=(2 n+1) x+\frac{4 n+3}{2 n x+\eta s}+\frac{4 n+5}{2(n+1) x+\eta s}
$$

and

$$
b_{n}=\frac{16(n+1)(n+2)}{[2(n+1) x+\eta s]^{2}} \text {. }
$$

The scalar recurrence Eqs. (B1)-(B3) may also be recast in the form of the matrix three-term recurrence relation (A2), viz.,

$$
\begin{aligned}
(\eta s & \left.-\mathbf{Q}_{n}\right) \widetilde{\mathbf{C}}_{n}(s)-\mathbf{Q}_{n}^{+} \widetilde{\mathbf{C}}_{n+1}(s)-\mathbf{Q}_{n}^{-} \widetilde{\mathbf{C}}_{n-1}(s) \\
& =\delta_{1, n} \eta \mathbf{C}_{1}(0) \quad(n \geqslant 1),
\end{aligned}
$$

where $\widetilde{\mathbf{C}}_{0}(s)=\mathbf{0}$,

$$
\widetilde{\mathbf{C}}_{n}(s)=\left(\begin{array}{c}
\widetilde{c}_{n-1}^{2,0}(s) \\
\widetilde{c}_{n-1}^{2,1}(s) \\
\widetilde{c}_{n-1}^{2,2}(s)
\end{array}\right) \quad(n \geqslant 2)
$$

[1] P. Debye, Polar Molecules (Chemical Catalog, New York, 1929, reprinted by Dover, New York, 1954).

[2] W. T. Coffey, Yu. P. Kalmykov, and S. V. Titov, J. Chem. Phys. 116, 6422 (2002).

[3] R. Metzler and J. Klafter, Adv. Chem. Phys. 116, 223 (2001).

[4] W. T. Coffey, Yu. P. Kalmykov, and S. V. Titov, Phys. Rev. E 65, 032102 (2002).

[5] E. P. Gross, J. Chem. Phys. 23, 1415 (1955).

[6] R. A. Sack, Proc. Phys. Soc. London, Sect. B 70, 402 (1957).

[7] R. A. Sack, Proc. Phys. Soc. London, Sect. B 70, 414 (1957).

[8] M. W. Evans, G. J. Evans, W. T. Coffey, and P. Grigolini, Molecular Dynamics and Theory of Broadband Spectroscopy (Wiley Interscience, New York, 1982).

[9] E. Barkai and R. S. Silbey, J. Phys. Chem. B 104, 3866 (2000).

[10] R. Carmichael, A Treatise on the Calculus of Operations (Longmans Green, London, 1855).

[11] E. C. Titchmarsh, An Introduction to the Theory of Fourier Integrals (Oxford University Press, London, 1937).

$$
\begin{aligned}
& \mathbf{C}_{1}(0)=\left(\begin{array}{c}
c_{0}^{2,0}(0) \\
0 \\
0
\end{array}\right) \\
& \mathbf{Q}_{n}^{-}=\left(\begin{array}{ccc}
0 & 2 & 0 \\
0 & 0 & 0 \\
0 & -4 n & 0
\end{array}\right) \\
& \mathbf{Q}_{n}^{+}=\left(\begin{array}{ccc}
0 & 0 & 0 \\
-3 n / 2 & 0 & 1 / 4 \\
0 & 0 & 0
\end{array}\right) \\
& \mathbf{Q}_{n}=\left(\begin{array}{ccc}
-2 x(n-1) & 1 / 2 & 0 \\
-6 n & -x(2 n-1) & 1 \\
0 & -(n-1) & -2 x(n-1)
\end{array}\right)
\end{aligned}
$$

The solution of Eq. (B5) is also given by Eq. (A3), where the column vector $\mathbf{C}_{1}(0)$ and the matrices $\mathbf{Q}_{n}^{-}, \mathbf{Q}_{n}^{+}$, and $\mathbf{Q}_{n}$ are defined by Eqs. (B6)-(B9).

[12] J. R. McConnell, Rotational Brownian Motion and Dielectric Theory (Academic, New York, 1980).

[13] W. T. Coffey, J. Chem. Phys. 93, 724 (1990).

[14] W. T. Coffey, J. Chem. Phys. 99, 3014 (1993).

[15] W. T. Coffey, Yu. P. Kalmykov, and J. T. Waldron, The Langevin Equation (World Scientific, Singapore 1996).

[16] J. L. Déjardin, Yu. P. Kalmykov, and P. M. Déjardin, Adv. Chem. Phys. 117, 275 (2001).

[17] S. Chapman and T. G. Cowling, The Mathematical Theory of Non-Uniform Gases, 3rd ed. (Cambridge University Press, London, 1970).

[18] J. H. Jeans, The Dynamical Theory of Gases, 4th ed. (Cambridge University Press, London, 1925, reprinted by Dover, New York, 1954).

[19] Handbook of Mathematical Functions, edited by M. Abramowitz and I. Stegun (Dover, New York, 1964).

[20] B. K. P. Scaife, Principles of Dielectrics (Oxford University Press, London, 1989).

[21] K. S. Cole and R. H. Cole, J. Chem. Phys. 9, 341 (1941). 
[22] G. Williams and D. C. Watts, Trans. Faraday Soc. 66, 80 (1970).

[23] B. K. P. Scaife, Complex Permittivity (English University Press, London, 1971).

[24] K. Weron and M. Kotulski, Physica A 232, 180 (1996).
[25] E. Lutz, Phys. Rev. E 64, 051106 (2001).

[26] G. E. Uhlenbeck and L. S. Ornstein, Phys. Rev. 36, 823 (1930).

[27] W. T. Coffey, J. Chem. Phys. 107, 4960 (1997). 